\title{
A Review on Equipped Hospital Beds with Wireless Sensor Networks for Reducing Bedsores
}

\author{
Lida Khaleghi
}

Ms.c of Health Information Technology, Medical Records Department- Salman Farsi Hospital- Sabz Abad BlvdBushehr-Iran

\author{
*Corresponding author \\ Lida Khaleghi, Ms.c of Health Information Technology, Medical Records \\ Department- Salman Farsi Hospital- Sabz Abad Blvd- Bushehr- Iran-P.O. \\ Box: 7518759416-Tel: +98-771-4542836; E-mail: lidakhaleghi@yahoo.com
}

Submitted: 03 Aug 2018; Accepted: 10 Aug 2018; Published: 17 Aug 2018

\begin{abstract}
At present, the solutions to prevent bed sore include using various techniques for movement and displacement of patients which is not possible for some patients or dangerous for some of them while it also makes problems for healthcare providers. On the other hand, development of information technology in health system including application of wireless sensor networks (WSN), has led to easy and quick service providing. It can provide a solution to prevent bed sore in motionless and disabled patients. Hence, the aim of this article was first; to introduce Wireless Sensor Networks in hospital beds and, second, to identify the benefits and challenges of implementing this technology. This study was a non-systematic review. The literature was searched for Wireless Sensor Networks to reduce and prevent bed sores with the help of libraries, databases (PubMed, SCOPUS, and EMBASE), and also searches engines available at Google scholar including during 1974-2014 while inclusion criterion was applied in English and Persian. In our searches, we employed the following keywords and their combinations: "wireless sensor network", "smart bed", "information technology", "Smart mattress", "bed sore" in the searching areas of titles, keywords, abstracts, and full texts. In this study, more than 45 articles and reports were collected and 37 of them were selected based on their relevancy. Therefore, identification and implementation of this technology will be a step toward mechanization of traditional procedures in providing care for hospitalized patients and disabled people. The Smart bed and mattress, either alone or in combination with the other technology, should be capable of providing all of novel features while still providing the comfort and safety features usually associated with traditional and hospital mattresses.
\end{abstract}

Keywords: Bed sore, Wireless sensor network, Advantage, Smart bed, smart mattress

\section{Introduction}

Decubitus ulcer is a common and serious problem for inpatients in hospital. When someone is in sleep mode, pressure of his body weight enters into certain points and in the long term, this will result in bruises and ultimately incurable wounds; therefore, hospital staff has to rotate these patients on their beds regularly to prevent it from happening. Due to the fact that prevention is more cost-effective than treatment and with the great cooperation of healthcare staff in the prevention of Decubitus ulcer, Decubitus ulcer is still as a significant problem for certain patients in hospital and society. Using wireless sensor network in hospital beds, which is known as smart beds, is an appropriate solution to prevent Decubitus ulcer and a way to forger the pressures of Decubitus ulcer in patients hospitalized for long time.

Bed sore is the third costly disorder after cancer and cardio-vascular diseases. In various studies, treatment costs have been estimated about $\$ 125-451$ for grade1 and 2 of bed sore and $\$ 1400-2300$ for grade 3 and 4 of bed sore. In Spain, $5.20 \%$, in the UK about 3.2\%, and in Netherlands more than one percent of total healthcare costs is spent on treatment of bedsore. In developed countries, the prevalence of bed sore varies from 3 to 30 percent and its incidence varies from $1-50 \%[1]$.

Annually, these wounds affect more than 1.3 million adults in the world. Bed sores can cause pain, depression, reduction of performance and independence, higher risk of infection and sepsis, all of which potentially lead to an increase in the duration of hospitalization [2]. Moreover, mortality rate caused by this disease has been high and can be effective at any age. According to a study titled "Prevention and Management of Pressure Ulcers in Primary and Secondary Care" conducted by Stans et al. (2014), even with hospital development and considerable healthcare resources, the prevalence of patients with bed sore shows a growth of $4.7-32.1 \%$ in hospitals and $22 \%$ in nursing homes [3].

In addition to heavy financial costs for healthcare institutes, bed sore causes consequences such as loss of institutes' credibility and waste of personnel's valuable time. In a study titled "Evidence-based Medicine: Pressure Sores" conducted by Cushing and Phillips in 2013, it was shown that annually with around 2.5 million bed sores in the United States, treatment cost of $\$ 11$ billion was estimated which represents a costly and labor-intensive challenge for healthcare system [4]. 
This condition is associated with pain, infection, and complications such as stress. The main groups at risk of bed sore include patients with spinal cord injuries, elderly, hospitalized patients with low mobility particularly those undergoing orthopedic surgery, and patients hospitalized in intensive care unit (ICU). The latter group is more susceptible than others because they are often motionless for long periods of time. Currently, there are mechanisms and devices some of which avoid focusing pressure on specific points (such as mattresses or cushions which distribute pressure or silicon foams) and some others increase pressure tolerance surface and apply uniform pressure to the human body [5]. Consequently, it helps pressurized points to receive blood and repair themselves.

Graves et al. (2005) conducted a research titled "Effects of Pressure Ulcers on Length of Hospital Stay" on 2000 patients during the years 2002-2003. The results indicated that patients suffering bed sore were hospitalized on average 4.31 days more than the time expected at reception [6].

Prevention is the most effective approach to solve this problem and high quality of nursing care is the key factor to resolve the problem. Preventive measures include:

1. Assessing the risk of bed sores progression;

2. Skin care and primary treatment such as skin health;

3. Use of pressure-reducing supportive surface such as wavy mattress and smart beds;

4. Training [7].

Moreover, the incidence of bed sore is influenced by predisposing potential factors which are placed in two groups of internal and external factors. Internal factors include malnutrition, reduction of movement and activities, aging, incontinence, skin moisture, mental status, and medication. External factors which are known to be effective in creating bed sore include pressure, friction, and tensile force applied by contact devices [8].

Identifying patients who are at risk of bed sore is crucial for effective treatment [9]. Researchers showed that identifying personal factors effective in the operation such as sense of individual responsibility, and organizational factors affecting current practices such as collaboration methods between nurses and nursing assistants is necessary to implement effective changes in a particular environment $[10]$.

Some experts believe that clinical judgment is the only reliable method to identify patients at risk while others believe that professional judgments are subjective criteria and risk assessment instruments are objective criteria which can identify patients at risk [11]. When nurses use complete, reasonable, and codified information obtained from risk assessment instruments, they lead to completely precise, specific, and defined patients' care instructions. At present, one of the most common methods for nurses and healthcare providers for prevention of these wounds is to turn and move patients from side to side every two hours. This allows recovering parts of the body. Therefore, with development of each bed sore, nursing staff work load increases [12].

In a research titled "Frequent Manual Repositioning and Incidence of Pressure Ulcers among Bed-bound Elderly Hip Fracture Patients" conducted by Rich et al. (2010), it was noted that only around 66\% of patients have received this treatment regularly which has been probably due to labor shortage in nursing. Also, this is a justifiable reason for back pain in healthcare workers [13]. Today, various technology developments I health has led to improvement of the quality and speed of service providing to patients [14].

Wireless sensor network is one of these technologies referring to a set of sensors connected wirelessly and all sensing a certain phenomenon. The number of these sensors can be very high and they can be also scattered over a wide domain. The sensors of this sensor can be annually placed and fixed at the desired locations or they can be randomly distributed in the desired location. The main purpose in these networks is primarily collection of information and then further network life $[15,16]$.

Therefore, with regard to issues and problems discussed, establishment of a smart bed can be a solution to these problems. In order to enhance bed capabilities and physical features of smart beds, smart areas are created from combination of sensor network, information devices, and computer control; the beds are capable to generate waves and signals for mobility and increasing blood flow in hospitalized patients while they prevent bed sore and lesions resulting from hospitalization. Hence, the aim of this article was first; to introduce Wireless Sensor Networks in hospital beds and, second, to identify the benefits and challenges of implementing this technology.

\section{Methods}

This study was a non-systematic review. The literature was searched for Wireless Sensor Networks to reduce and prevent bed sores with the help of libraries, databases (PubMed, SCOPUS, and EMBASE), and also searches engines available at Google scholar including during 1974-2014 while inclusion criterion was applied in English and Persian. In our searches, we employed the following keywords and their combinations: "wireless sensor network", "smart bed", "Smart mattress", "information technology", and "bed sore" in the searching areas of title, keywords, abstracts, and full texts. In this study, more than 45 articles and reports were collected and 37 of them were selected based on their relevancy.

\section{Results}

Introducing wireless sensor networks in hospital beds

Types of wireless networks

- Infrastructure-based networks: To implement desired wireless networks, one or more central concentrator devices or access points (AP) are applied. The task of AP is to communicate on the network.

- Wireless LANs: Usually, radio links [11] or infrared radiation is used. They create high flexibility in the range in which the possibility is placed and have less bandwidth compared to wired networks.

- AD hoc networks: These wireless networks contain a set of distributed nodes which communicate wirelessly with each other. The most important feature of these networks is a dynamic and variable typology due to nodes mobility. [15] Wireless sensor networks are one of these networks.

A sensor network is composed of a large number of sensor nodes which are widely distributed in an environment and collect information from the environment. The position of sensor nodes is not necessarily predetermined. Such feature makes it possible to leave them in hazardous and inaccessible situations [15].

There are several terms in sensor network: 
Sensor: A device that detects objects, events or the value of a physical quantity and converts it into an electrical signal. There are several types of sensors such as sensors of temperature, pressure, humidity, accelerometer, and magnetometer.

Operate: With electrical simulation performs a particular action such as opening or closing a valve or switching off a switch.

Sensor node: It is a node which only includes one or more sensors.

Operate node: It is a node which only includes one or more operates.

Sensor network: It is a network which only includes sensor nodes. This network is a particular type of work/sense node and is applied in applications in which the purpose is collection of information and investigation of a phenomenon such as tornados.

Operate/sensor field: the work area in which work/sense nodes are distributed.

Sink: It is a node which is responsible for data collection and establishes the connection between work/sense nodes and task manager node.

Work/sense net: A network of excessive number of nodes where each node can have a number of sensors and operates [16]. These nets take the information of the environment through the sensors and react through operates. Communication between nodes is wireless.

Each node operates independently and without human intervention. It is physically very small and limited in processing power, memory capacity, and power source. These restrictions create problems which are the origin of many research discussions in this field. Information is collected by sink and commands are distributed through sink (figure1) [17].

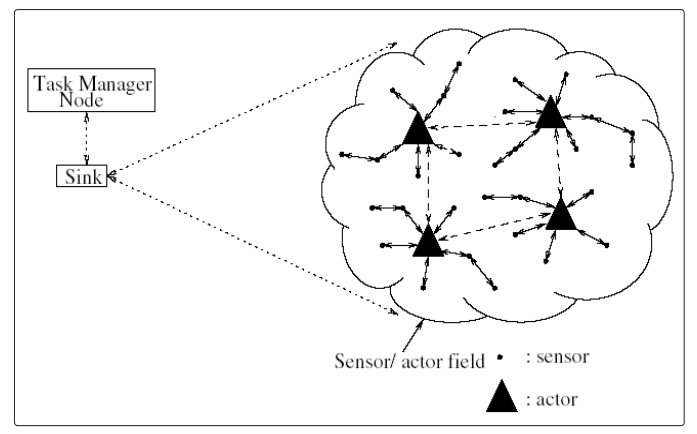

Figure1: General structure of work/sense net [17]

Task manager node: It is a node through which the person as a user or network administrator communicates with the network. Queries and control commands are sent from this node to the network and collected data is returned to it [17].

Automated structure: Sensors which detect an event or a phenomenon and send the received data to operate node to process and react appropriately. Adjacent operate nodes, coordinating together, decide and operate. In fact, there is no central control and decisions are made locally (figure2) [17].

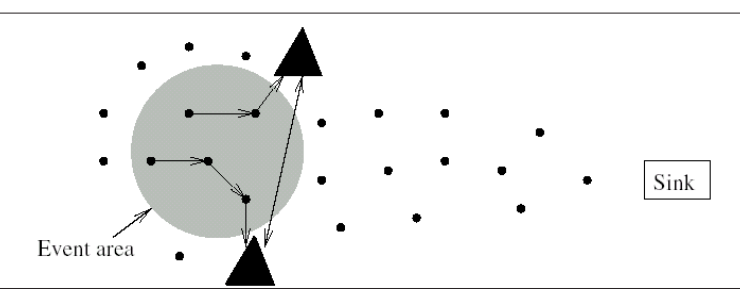

Figure 2: Automated Structure [17]

Semi-automatic structure: In this structure, data is conducted by nodes to the sink and commands are distributed to operate node through the sink (figure3) [17].

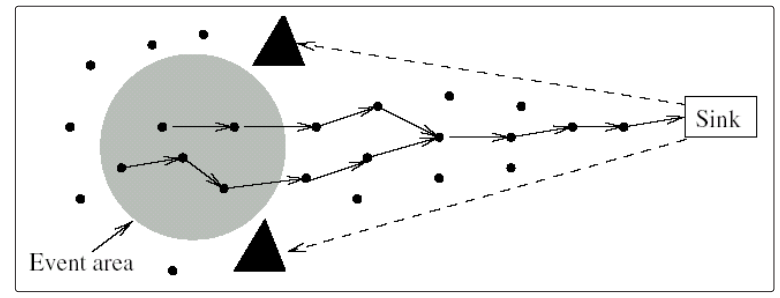

Figure 3: Semi-automatic structure [17]

Operate/sensor node: it is a node, equipped with sensor and operates.

Internal structure of each node includes operate/sensor field, data processing field, sender/receiver wireless, and power source. Data processing field consists of a microprocessor and a memory with a limited capacity. It takes data from the sensor, does limited processing depending on the application, and sends through the sender. Field processor performs coordination management with other nodes in the network. Sender/receiver communicates between node and network [18].

Sensor unit includes a set of sensors and analog to digital convertor; it takes analog data from the sensor and delivers it digitally to processor. Operate unit consists of operate and analog to digital convertor; it takes digital commands from the processor and delivers them to the operate. Field energy generator provides consumption power of all sectors which is often a limited power battery (figure 4) [19].

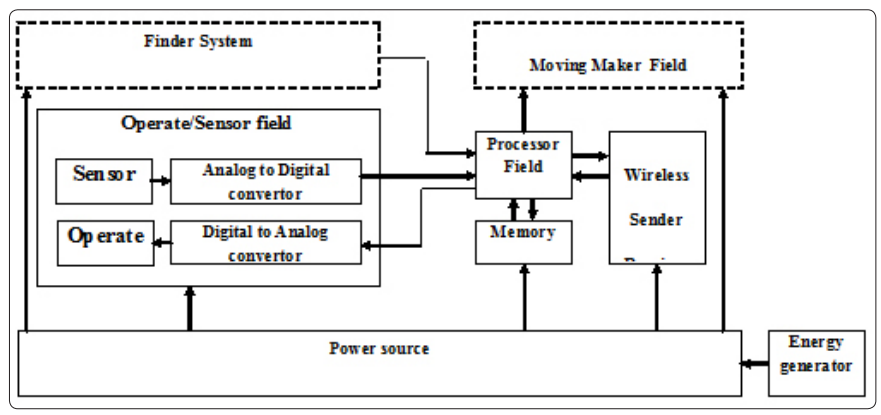

Figure 4: Internal structure of operate/sensor node [19]

Energy source restriction is one of the major constraints which affect everything in designing work/sense net. Beside this section, a unit may exist for energy such as solar cells; there is a unit for moving maker in mobile nodes. Finder detects the node physical location. Routing techniques and sensing functions require highprecision location information. One of the main benefits of work/ 
sense network is the ability to manage communication between the nodes on the move [19].

\section{Applications of wireless sensor networks}

WSN applications are divided into three categories of military, commercial, and medical applications. Systems of communication, command, reconnaissance, monitoring, smart minefield, and smart defense systems are of the military applications of these networks. Medical care applications include systems of care for patients with diabetes who do not have a caregiver, smart environments for elderly people, communication networks among practitioners and hospital staff, and patients' monitoring. Commercial applications include a wide range of applications such as security systems to detect and deal with theft, fire (in forest), detecting environmental pollution like chemical, microbial, and nuclear pollution, tracking systems, maintaining and control of vehicles and traffic, controlling the quality of industrial products, studying natural phenomena such as tornado, earthquake, and flood, and also research on particular species of animals and plants. In some applications, work/sense net is applied as a group of small robots which work together to perform a specific activity [20].

Technology application in healthcare is considered as the most important issue to improve the quality of healthcare and researchers have indicated that it is the best instrument to improve and promote quality of patients' care and ultimately leads to promotion of community's health.

Advantages and challenges of implementing wireless sensor network

In addition to what already stated about WSN, implementation of these networks has also a series of features, disadvantages, and challenges the most important of which is summarized in table1.

Table 1: Advantages and challenges to implement wireless network sensors

\begin{tabular}{|l|l|}
\hline Advantages & Disadvantages and challenges \\
\hline $\begin{array}{l}\text { Dependence on the application } \\
{[15]}\end{array}$ & Hardware constraints $[16,17]$ \\
\hline $\begin{array}{l}\text { Interaction with the environment } \\
{[15,16]}\end{array}$ & Typology [17, 19] \\
\hline Scalability [15] & Reliability of use [16, 18] \\
\hline Energy [16, 19] & Scalability [18] \\
\hline Self-configuration [16. 18] & Environmental conditions [16, 18] \\
\hline $\begin{array}{l}\text { Reliability and quality of service } \\
{[16.19]}\end{array}$ & Cost [19] \\
\hline Data-driven [17, 18] & $\begin{array}{l}\text { Increasing network life time [16, } \\
20]\end{array}$ \\
\hline Simplicity [15, 18] & $\begin{array}{l}\text { Power consumption of the nodes } \\
{[17,20]}\end{array}$ \\
\hline
\end{tabular}

Unfortunately, information systems in many countries are inadequate to provide the required management support [21]. It is clear that an information system can influence decision making only if the information is reliable, related, and available to decision makers at appropriate time [22]. Reliable and updated information can affect destructive factors and prevent them [23]. Information systems play an important role in continuity of activities related to healthcare, management, and planning of health services. Such systems can be considered like an instrument which facilitates processing, storing, and transferring of data in health management relations.

Smart materials and their application

Applications of smart materials in clothing

The first generation of smart materials can be termed the generation of electronic clothes, since a number of electronic components have been added to conventional clothes. The first successful step to make them wearable was taken by an American company in 1990s which supplied clothing for army [24]. Since birth, the first application interface for body is soft and reassuring materials of the fabric. Fabric usually covers more than $80 \%$ of skin and in the new generation of textile materials, new intuitive and interactive functions have been applied to implement personal security systems, measurements, transmission, and expansion through textile technology [25]. To achieve this clothing, electrical properties and calculations with traditional mechanical properties have been used which are not comparable to fabric normally used to produce ordinary clothes. A German company invented a miniature MP3 player which can be placed in the clothing. Its completed design consisted of a central microchip, a phone, a battery, a special card to download music, and an internal communication system through conductive sinew fabric. Waterproof packages protect all components against water. Nevertheless, components added to clothes always created problems when being worn and the communication between clothes and components always caused trouble [24, 25].

Application of smart materials in textile

In the next generation, it was tried to use textile materials as the components [25]. English company, Eleksen, has provided a new technology which enables them to combine sensors and sensor switches with textile warp and woof. These smart fabrics with flexibility and folding features can be washed and sewn. This property will be applied in special clothing, making dolls, mobile phone covers, aircraft seats, and hospital bed mattresses [26].

These fabrics are a combination of conductive fibers and conventional textile fibers which have been made of two exterior plastic electric layers between which a woven layer of conductive fibers is placed. The voltage to measure the suppliers is guided by batteries; when pressure is exerted on the outer layers (such as finger pressure), sensors' voltage changes. A sensor which detects the change can change the exerted pressure according to the conditions [26, 27]. Moreover, this company has manufactured a fabric which is sensitive to hand contact and the smart fabric of this company called Elektext, is composed of three layers of fabric. The layered feature of this fabric makes it possible to transfer generated electric charge by computer software to other layers after being touched by hand [27].

A flexible keyboard for Personal Digital Assistant (PDA) which can be applied in bed sensors in hospitals is another product of this company [28].

\section{Electro-rheological fluid (ERF)}

Another category of smart materials is electro-rheological fluid which is controlled by applying a potential difference. These fluids frozen by applying an electrical voltage are called smart fluid. Until recently, their naming didn't seem so meaningful. However, now some engineers comment that smart fluids will be the initiator of the third era of the machine. Smart fluids are not new materials and are not so smart. It is more than 70 years that scientists have found that 
some liquids are converted to solid by applying electrical voltage. This phenomenon is called Electro-Rheological Effect. ERF is smart in the respect that when applied high voltage, it is converted into gelatinous solid called viscosities. These fluids can be used in smart beds. A network of plates equipped with ERF is placed in these beds and electrical potential difference is applied to different locations at appropriate times. This way we increase the viscosity of these locations compared to other locations to transfer the point bearing pressure to desired places or distribute the pressure and prevent bed sore in patients' skin [29].

Since in other solutions, patients or people who help them have no perception of the pressure value and distribution, the changes may lead to patients' more critical conditions. Therefore, the main advantages of applying these fluids in bed design include the ability to detect pressure points, measuring pressure field, no need to move the patients, and controlling patients' recovery terms by computer while there is no need for constant presence of doctors and nurses [30].

Well sense company in USA, is a leader in continuous bedside pressure mapping technology, is a mobile health company dedicated to patient safety throughout the continuum of care. The company's founders and management bring a strong track record of innovating and commercializing breakthrough technologies in consumer electronics, patient safety and monitoring. Well sense is a privately held company. For long-term hospital patients or people who are otherwise bedridden, bedsores can be a major problem. Technically known as Decubitus ulcers, they form when one area of the skin is subjected to too much prolonged pressure. In order to keep them from occurring, hospital staff regularly turns patients over in their beds. The MAP (monitor, alert, protect) System is designed to aid those caregivers, by providing them with real-time imagery of the pressure points on the patient's body. Made by medical tech firm Well sense, MAP consists of two main parts: a pressure-sensing mat that is placed on an existing mattress, and a bedside monitor. Thousands of sensing points within that mat continuously register how much pressure is being exerted upon them by the patient, and relay that information to the monitor. There, a corresponding pressure distribution map of the patient's body is displayed, indicating highand low-pressure areas. Using that information, staff can make sure to relieve pressure in the crucial places when repositioning the patient. A countdown also appears on the screen, indicating the amount of time left before the next repositioning will be required. The general idea of using pressure-sensing mattress pads to minimize the occurrence of bedsores isn't particularly new, in and of itself. In most cases, however, it's done to obtain a single snapshot-style reading, which is then used as a reference. MAP is reportedly unique in that it provides continuous, real-time feedback. Well sense certainly isn't the only group working at alleviating bedsores. Swiss research group Empa has created sheets that produce fewer points of contact with the skin, along with a bed that repositions the patient by moving the mattress. Multiple clinical studies using MAP and without MAP in both hospitals ICU that demonstrated significantly reduced and in some cases down it to zero, and economic benefits ranging from $\$ 125,000$ to 650,000 in saving per ICU over 6 months (figure 5) [31].

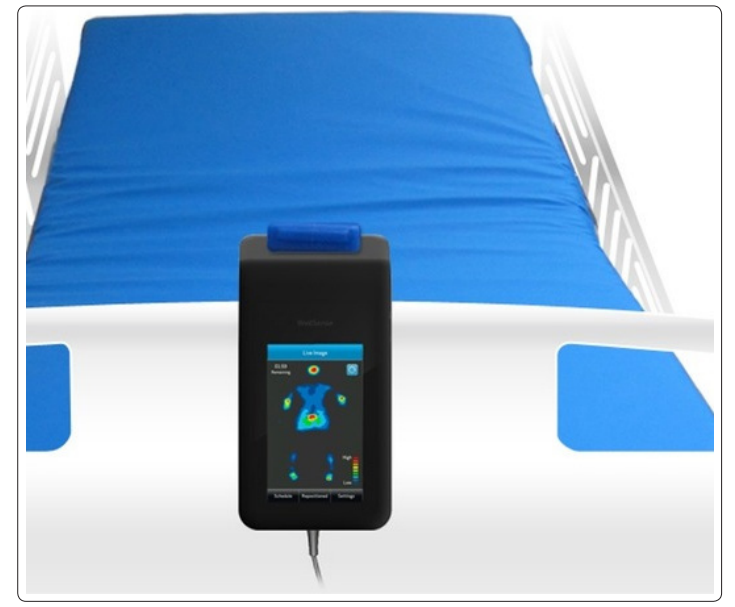

Figure 5: the MAP monitor and pad [31]

The pilot study showed the smart bed system recorded a total of 1,037 in bed alerts; 1,033 bed exit alerts (with manual adjustments making up the difference in number). Within that period, there were zero reported and documented falls. Alerts were set for the two-hour turning of bedbound residents. The Touch-free Life Care (TLC) system recorded that staff performed 576 documented turns on schedule, and the system recorded that 318 scheduled turns were missed. This turn performance data were used by the staff to improve their timely performance of turns. Within the study period, the facility did not report any development of new pressure ulcers and, in fact, reported the improvement in staging of one resident's pressure ulcer. The system also gathered heart rate and respiratory rate trends for all residents, rest time and time in bed per day, and the identification of motion in the bed as it correlated to documented turn events (figure 6) [32].

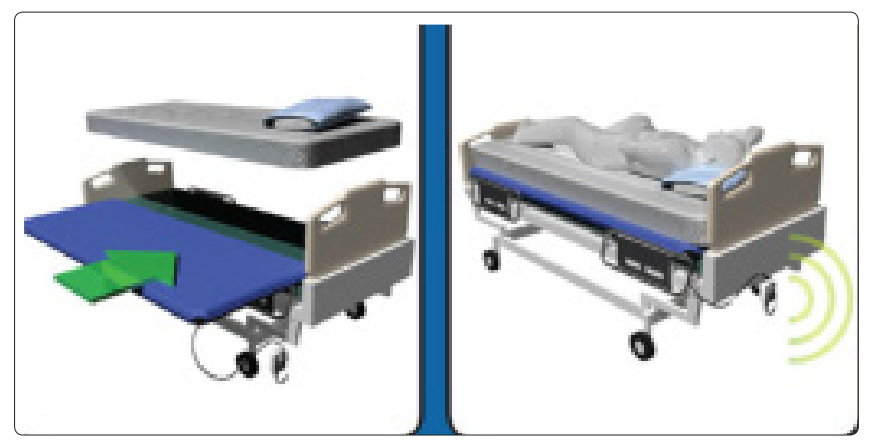

Figure 6: The BAM Labs Smartbed mat slides under the mattress [32]

\section{Discussion}

In this regard, results of Chenu et al. study in 2013 entitled "an innovative embedded device for pressure ulcer prevention" showed that the Decubitus ulcer prevention device for patients with spinal injury, which has the size of cigarette box and is embedded in a wheelchair, can be effective in preventing them. The device sensor is one hundred percent textile, washable and inexpensive. The device function is to announce acquired alarm via watch or SMS in Smartphone before symptoms develop Decubitus ulcer and by measuring the pressure between the seat mattress and patient's hip [33]. 
Yousefi et al. in their study in 2011 entitled "a smart bed platform for monitoring \& ulcer prevention" defined hospital smart bed as a bed with a sensor network, information devices, tile like surface and computer control. This bed function was to analyze data by creating touch and pressure on surface sensors and creating irritation and then announce acquired alarm to the nurse to prevent ulcer pressure. Thus, the nurse spends more time for assessing events adverse and outcomes and patient's care instead of rotating patient every moment [34].

Since Decubitus ulcer is a big problem in health care of patients with spinal injuries and these patients have no feeling for changing their status in comparison with healthy people, using a mattress with sensor is useful to diagnose the patient's problem and patient's tactile response. Using such system is useful in rehabilitation and is suitable for preventing Decubitus ulcer.

In a study done by Verbunt et al. in 2010 entitled "tactile feedback for the prevention of Decubitus ulcers", a technology is defined which is able to diagnose patient's inappropriate status and present an instruction to change his status. Changing status resulted in less risk of Decubitus ulcer. This device includes a belt that receives a simple tap to a strong pressure as a signal and this signal announces changing patient's natural status to patient and health care providers [35].

Wireless sensor networks are increasingly changing for telemedicine applications, monitoring patients, both in clinical conditions and at home. Using them will reduce user's discomfort and expenses, and increase mobility. Using smart systems and wireless sensor networks, depending on users' needs, will also collect information regarding users and their environment [36-37].

\section{Conclusion}

Today, life cannot be imagined without wireless communication. Health care providers are very mobile, and the adoption of enhanced wireless technology by health care organizations, especially when harnessed properly, can help to improve, even automate, patient care and monitoring, save costs, and reduce staff injuries. It is indisputable that computer networks are commonplace in health care organizations and in some places are indispensable. However, most of the computer devices and monitoring devices are connected through the use of wires; this usually means that their use is limited to a fixed place. A Smart Mattress eliminates many of the wires associated with current methods and allows for greater flexibility in patient monitoring. The Smart Mattress, either alone or in combination with the other technology, should be capable of providing all of novel features while still providing the comfort and safety features usually associated with traditional and hospital mattresses. For example, the Smart Mattress should be designed to reduce the potential for pressure ulcers.

In fact, the power of wireless sensor networks is the result of applying a large number of tiny nodes which are capable to be organized and applied in various cases such as simultaneous routing, monitoring environmental conditions, and monitoring health of structures or equipment of a system. Also, technology progress and creation of smaller and smaller circuits have made application of wireless circuits possible in most electronic devices. Therefore, applying these wireless circuits in hospital beds is helpful for elderly people, long-term hospitalized patients or patients without movement, and also for hospital staff to monitor patients' treatment and to prevent bedsore. However, widespread use of this technology requires focusing on related systems and help of professional experts.

Sensor nets have provided a new range of applications, particularly in the field of monitoring chronic diseases. In heart diseases, smart sensor nodes can be attached on patients' bodies and prevent death which occurs due to heart failure. In cancer detection, sensor nodes are placed in suspected location and measure the amount of nitric acid produced by these glands. People with Alzheimer are not aware of time and location where they are and always act abnormally. These networks inform person's neighbors and relatives of any unusual situations. Monitoring glucose level is performed by a sensor attached to patient's body and it measures blood glucose level. To monitor epilepsy, sensors detect the seizures before they occur by monitoring brain activities in these patients. Patients' vital signs such as heart rate, respiratory rate, and body temperature can be monitored permanently and continuously by sensors. Thus, patients' health status can be supervised during the day and even during sleep or exercise.

Imagine a person who is at irregular heart rhythm. In some cases, the irregularities in patient's heart rate are severe and slow heart beat for a long time while revascularization does not occur completely and the brain injury happens. Therefore, these patients should be supervised permanently so that if there is an irregularity, necessary operations can be performed. Normally, it is necessary to hospitalize the patient and take ECG for a time, but using WSN, patients can be monitored remotely all the time without the need to be hospitalized and confined to hospital beds.

In all, according to care method and costs of the care, patient care is very important for caregivers and also for patients and their families. Finding the best and most economic method of patient care has engaged the minds of many experts in various fields of medical services or IT and electronic engineering. With application of smart materials or micro-sensors in medical equipment and attaching those to patients' as well as physicians these conditions can be controlled to a great extent and standard care can be provided for patients.

\section{Authors' Contribution}

SA contributed to the conception and design of the work, conducting the review study, preparing and revising the manuscript, approval of the final version of the manuscript, and agreed for all aspects of the work. LKh contributed to the conception and design of the work, revising the manuscript, approval of the final version of the manuscript, and agreed for all aspects of the work.

\section{Acknowledgments}

Support \& Source of funds: This article resulted from part of master thesis in the School of Medical Management \& Information Sciences, Isfahan University of Medical Sciences, Iran.

Conflict of Interest: None declared.

\section{References}

1. 1-Lisett S, Haalboom JR, Bousema JRE, Algra A, Grypdonck MH, et al. (2002) Prospective Cohort Study of Routine Use of Risk Assessment Scales for Prediction of Pressure Ulcers. BMJ 325: 779-785. doi: http://dx.doi.org/10.1136/bmj.325.7368.797. [ Last accessed on January 25, 2015]. 
2. Soozani A, Khosravi A, Pourheydari M, Montazeri A (2011) Using Braden and Waterlow Scales to Predict Pressure Ulcer: A Comparative Study. Journal of Knowledge \& Health Faculty of Nursing and Midwifery, Shahroud University of Medical Sciences 5: 43 48. Http: //dx.doi. org/10.1234/knh.v5i4.161. g113.

3. Stansby G, Avital L, Jones K, Marsden G (2014) Prevention and Management of Pressure Ulcers in Primary and Secondary Care: Summary of NICE Guidance. BMJ 348. Doi: http://dx.doi. org/10.1136/bmj.g2592

4. Cushing CA, Phillips LG (2013) Evidence-based Medicine: Pressure Sores. Journal of Plastic and Reconstructive Surgery 132: 1720-1732. Doi: 10.1097/PRS.0b013e3182a808ba.

5. Santamaria N, Liu W, Gerdtz M, Sage S, McCann J, et al. (2013) The Cost-Benefit of Using Soft Silicone Multilayered Foam Dressings to Prevent Sacral and Heel Pressure Ulcers in Trauma and Critically Ill Patients: a Within-Trial Analysis of the Border Trial. International Wound Journal. Doi: 10.1111/ iwj. 12160.

6. Graves N, Birrell F, Whitby M (2005) Effect of pressure ulcers on length of hospital stay, Infect Control Hosp Epidermal 26: 293-297. Doi: http://dx.doi.org/10.1234/knh.v5i4.161.

7. Pancorbo-Hidalgo PL, Garcia-Fernandez FP, Lopez-Medina IM, Alvarez-Nieto C (2006) Risk Assessment Scales for Pressure Ulcer Prevention; a Systematic Review. J Adv Nurse 54: $94-$ 110. Doi : 10.1111/j.1365-2648.2006.03794.x

8. Reddy M, Gill S, Rochon P (2006) Preventing Pressure Ulcers; a Systematic Review. JAMA 296: 974-984. Doi:10.1001/ jama.296.8.974.

9. Kottner J, Dassen T (2010) Pressure Ulcers Risk Assessment in Critical Care, Integrator Reliability and Validity Studies of Braden and Waterlow Scales and Subjective Rating in Two Intensive Care Units, Int J Nurs Stud 47: 671-677. Doi: 10.1016/j.ijnurstu.2009.11.005.

10. Dellefield ME, Magnabosco JL (2014) Pressure Ulcer Prevention in Nursing Homes: Nurse Descriptions of Individual and Organization Level Factors. Journal of Geriatric Nursing 35: 97-104. Doi: 10.1016/j.gerinurse.2013.10.010.

11. Gould D, Goldstone L, Kelly D, Gammon J (2004) Examining the Validity of Pressure Ulcer Risk Assessment Scales; a Replication Study. Int J Nurs Stud 41: 331-339. Doi: http:// dx.doi.org/10.1016/j.ijnurstu.2003.10.005.

12. Asimus M, Maclellan L, Li PI (2011) Pressure Ulcer Prevention in Australia: the Role of the Nurse Practitioner in Changing Practice and Saving Lives. International Wound Journal 8: 508-513. Doi: 10.1111/j.1742-481X.2011.00824.X.

13. Rich SE, Margolis D, Shardell M, Hawkes WG, Miller RR, et al. (2011) Frequent Manual Repositioning and Incidence of Pressure Ulcers among Bed-Bound Elderly Hip Fracture Patients. Wound Repair and Regeneration 19: 10-18. http:// dx.doi.org/10.1111\%2Fj.1524-475X.2010.00644.x

14. Ajami S, Ketabi S (2011) Saghaeiannejad-Isfahani S, Heidari A. Readiness Assessment of Electronic Health Records Implementation. Acta Informatica Medica 19: 224-227. Doi: 10.5455/aim.2011.19.224-227

15. Santi P (2005) Topology Control in Wireless Ad hoc and Sensor Networks. Journal of ACM Computing Surveys. USA. New York 37: 164-194. Doi>10.1145/1089733.1089736

16. Tang M, Bai J, Li J, Xin Y (2014) Distributed Optimal Power and Rate Control in Wireless Sensor Networks. Scientific World Journal 580854. Doi:10.1155/2014/580854.
17. Wen CY, Chen YC (2009) Dynamic Hierarchical Sleep Scheduling for Wireless Ad-hoc Sensor Networks. Sensors (Basel)Journal 9: 3908-3941. Doi:10.3390/s90503908

18. Pilloni V, Atzori L (2011) Deployment of Distributed Applications in Wireless Sensor Networks. Sensors (Basel) Journal 11: 7395-7419. Doi:10.3390/s110807395.

19. Huang CF, Tseng YC (2003) The Coverage Problem in a Wireless Sensor Network. Wireless Sensor Networks and Applications 115 - 121. Doi>10.1145/941350.941367.

20. Yick J, Mukherjee B, Ghosal D (2008) Wireless sensor network survey. Computer Networks Journal 52: 2292-2330. DOI: 10.1016/j.comnet.2008.04.002.

21. Ajami S (2013) The Role of Earthquake Information Management System to Reduce Destruction in Disasters with Earthquake Approach. In Dr. John Tiefenbacher (Ed), Approaches to Disaster Management - Examining the Implications of Hazards, Emergencies and Disasters. Croatia: INTECH 131-144.

22. Ajami S, Fatahi M (2009) The Role of Earthquake Information Management Systems (EIMSs) in Reducing Destruction: A Comparative Study of Japan, Turkey and Iran". Disaster Prevention and Management 18: 150-161. Doi. 10.1108/09653560910953225.

23. Ajami S (2012) A Comparative Study on Earthquake Information Management Systems (EIMS) in India, Afghanistan and Iran. Journal of Education and Health Promotion 1: 27-34. Doi: 10.4103/2277-9531.99963.

24. Lymberis A, Paradiso R (2008) Smart fabrics and interactive textile enabling wearable personal applications: R\&D state of the art and future challenges. Engineering in Medicine and Biology Society (EMBC) 5270-5273. Doi: 10.1109/ IEMBS.2008.4650403.

25. Jacq C, Maeder T, Ryser P (2009) Load Sensing Surgical Instruments. Journal of Materials Science 1: S223-227. Doi: 10.1007/s10856-008-3533-z.

26. McLellan Ch. Eleksen Wireless Fabric Keyboard: a First look. The ZDNet Newsletters 2006 July 17; Sect. A: 2. Available from: URL: http://www.zdnet.com/eleksen-wireless-fabrickeyboard-a-first-look-3039278954/ . [ Last accessed on January $25,2015]$.

27. Winslow R. ElekTex Smart Fabric. 2007 May[ cited 2014 May 17]. Available from: URL:http://www.crunchwear.com/elektexsmart-fabric/.[ Last accessed on January 25, 2015].

28. Ajami S, Torabian F (2013) Mobile Technology in Healthcare. J Inform Tech Soft Engg S7: e006. Doi:10.4172/2165-7866. S7-e006

29. Arguelles J, Martin HR, Pick R (1974) A Theoretical Model for Steady Electro viscous Flow between Parallel Plates. Journal of Mechanical Engineering Science 16: 232-239. Doi: 10.1243/ JMES JOUR 197401604202.

30. Bullough WA (1991) Electro-rheological fluids: an introduction for biomedical applications. Journal of Biomedical Engineering 13: 234-238. Doi: 10.1016/0141-5425(91)90133-R.

31. Coxworth B. MAP System continuously monitors patients for bedsores. August 15, 2013 . Available from: http://www.gizmag. com/map-pressure-mapping-bedsores/28723/

32. Fernandez-Luque FJ, Zapata J, Ruiz R. A system for ubiquitious fall monitoring at home via a wireless sensor network. 32nd Annual International $\mathrm{C}$ onference of the IEEE EMBS. Buenos Aires, Argentina, August 31-September 4, 2010.

33. Chenu O, Vuillerme N, Bucki M, Diot B, Cannard F, et al. (2013) TexiCare: an innovative embedded device for pressure 
ulcer prevention. Preliminary results with a paraplegic volunteer. Journal of Tissue Viability 22: 83-90. doi: 10.1016/j. jtv.2013.05.002. Available from: http://www.ncbi.nlm.nih.gov/ pubmed/?term $=$ smart+sensor+for+bedsore. [Last accessed on January 25, 2015].

34. Yousefi R, Ostadabbasi S, Faezipour M, Nourani M, Ng V, et al. A Smart Bed Platform for Monitoring \& Ulcer Prevention. in: 4th International Conference on Biomedical Engineering and Informatics (BMEI). Antalya, Turkey 2011. [Last accessed on January 25, 2015].

35. Verbunt M, Bartnech C (2010) Sensing Senses: Tactile Feedback for the Prevention of Decubitus Ulcers. Journal of Applied Psychophysiology and Biofeedback 35: 243-250. DOI 10.1007/ s10484-009-9124-z. Available from: http://link.springer.com/ article/10.1007/s10484-009-9124-z.
36. Zubiete ED, Luque LF, Rodríguez AV, González IG (2011) Review of wireless sensors networks in health applications. Conf Proc IEEE Eng Med Biol Soc. doi: 10.1109/ IEMBS.2011.6090510. Available from: http://www.ncbi.nlm. nih.gov/pubmed/22254675. [Last accessed on January 25, 2015].

37. Ajami S, Khaleghi L (2015) Hospital Beds Wireless Sensor Network and Reducing Decubitus. JRMS In press.
Copyright: (C2018 Lida Khaleghi. This is an open-access article distributed under the terms of the Creative Commons Attribution License, which permits unrestricted use, distribution, and reproduction in any medium, provided the original author and source are credited. 